Proceedings of the 43rd "Jaszowiec", International School and Conference on the Physics of Semiconductors, Wisła 2014

\title{
Low Temperature Processing of Nanostructures Based on II-VI Semiconductors Quantum Wells
}

\author{
M. Majewicz ${ }^{a}$, D. ŚnieżeK ${ }^{b}$, T. Wojciechowski ${ }^{a}$, E. Baran $^{b}$, P. NOWICKi $^{a}$, \\ T. WOJTOWICZ ${ }^{a}$ AND J. WRÓBEL ${ }^{b, a}$ \\ ${ }^{a}$ Institute of Physics, Polish Academy of Sciences, al. Lotników 32/46, 02-668 Warszawa, Poland \\ ${ }^{b}$ Faculty of Mathematics and Natural Sciences, Rzeszów University, al. T. Rejtana 16A, 35-959 Rzeszów, Poland \\ We report the first results of electron beam lithography processes performed on polymethyl methacry- \\ late (PMMA) and hydrogen silsesquioxane (HSQ) resists, which have been pre-backed in vacuum at $T \leq 90^{\circ} \mathrm{C}$. \\ For such low temperature processing the lithographical resolution is reduced as compared to standard procedures, \\ however, the exposure contrast and adhesion to CdTe and HgTe substrates have been sufficient for the fabrication \\ of sub- $\mu \mathrm{m}$ quantum devices. Furthermore, the new method of electrical microcontact forming is proposed, based \\ on the local melting and annealing of an indium metal layer, performed with the application of accelerated electron \\ beam. The method has been tested for $\mathrm{CdTe} / \mathrm{CdMgTe}$ quantum wells using the lithography techniques, the expo- \\ sure parameters have been optimized by inspecting the morphology of annealed metal film via the in situ imaging.
}

DOI: 10.12693/APhysPolA.126.1174

PACS: 73.61.Ga, 73.63.Hs, 68.37.Hk, 62.23.St

\section{Introduction}

Modulation doped quantum wells made of II-VI materials possess unique properties as compared to their III-V counterparts. For example, CdMgTe/CdMnTe structures are important in constructions of novel spintronic devices [1], whereas $\mathrm{HgTe} / \mathrm{CdTe}$ heterostructures dominate the research of two-dimensional topological insulator phase $[2,3]$. There are, however, relatively few experimental studies, devoted to the properties of one- and zero-dimensional nanostructures fabricated of the above mentioned materials $[4,5]$ with the application of electron beam lithography (EBL).

One of the reasons is that II-VI semiconducting alloys are characterized by the high inter-diffusion coefficients and the low defect formation energies [6]. Therefore, in contrast to III-V compounds, relatively low temperatures of pre- and post-processing are required at all technological stages during the nanostructurization phase. For example, modulation doped $\mathrm{HgCdTe} / \mathrm{HgTe}$ quantum wells are typically grown at $180^{\circ} \mathrm{C}[7]$. For such growth temperature, HgTe inter-diffusion constant is high enough to blurr the interface thickness by the additional $\approx 1.8 \mathrm{~nm}$ after one hour of $180^{\circ} \mathrm{C}$ post-growth heating [6]. Hence, the upper limits of $85^{\circ} \mathrm{C}$ [2] or $100^{\circ} \mathrm{C}$ [4] are recommended for the $\mathrm{HgTe}$ quantum well substrates. Such low temperatures, however, are incompatible with the high resolution EBL resists like polymethyl methacrylate (PMMA, pre-backing temperature $180^{\circ} \mathrm{C}$ ) or hydrogen silsesquioxane (HSQ, $150^{\circ} \mathrm{C}$ ).

Furthermore, the strict temperature limitations pose a serious problem with thermal forming of electrical ohmic contacts, specially in the case of $\mathrm{CdMgTe} / \mathrm{CdTe}$ modulation doped quantum wells. For these materials the best results were obtained with macroscopic indium (In) contacts, which are burnt-in [8] or soldered with soldering iron [5] directly to the surface of large contact pads.
Clearly, such procedures are not fully reproducible and again, they are not compatible with e-beam lithography.

In this work we propose to overcome the above mentioned incompatibilities by pre-backing PMMA and HSQ resists in vacuum at temperature $T \leq 90{ }^{\circ} \mathrm{C}$. Additionally, we test the possibility of melting and annealing the lithographically defined indium micro-contacts locally, with the application of high-energy focused electron beam.

\section{Materials and results}

Polymethyl methacrylate (PMMA) is an organic polymer, commonly used in electron-beam lithography as a high-resolution positive tone resist [9]. Usually, PMMA is dissolved in chlorobenzene or anisole to create a casting solution, which is deposited onto substrates with spin-on process. After spinning, resist undergoes a pre-bake process, typically about one hour at $180^{\circ} \mathrm{C}$. The purpose of this stage is twofold. First, annealing removes micropores and voids within the polymer film [10]. Second, the casting solvent must be completely evaporated out, to improve the adhesion and strengthen the resistance against chemicals [11]. Therefore, the recommended bake temperature should be well above the solvent boiling point. Keeping this in mind we propose the following approach.

- Reduce the pre-backing temperature to $\leq 90{ }^{\circ} \mathrm{C}$. This value is within the recommended range for IIVI materials and close enough to the so-called glass transition, required to densify the polymer film [10].

- Reduce the pressure $p$ during the annealing process in order to lower the solvent's boiling point well below $90^{\circ} \mathrm{C}$ limit. From the Clapeyron-Clausius relation we have estimated that for chlorobenzene (which evaporates at $132{ }^{\circ} \mathrm{C}$ ) the boiling point lowers to $17^{\circ} \mathrm{C}$ already at $p=0.01 \mathrm{~atm}$. Therefore, only a moderate pressure reduction is necessary. 
We have performed the proposed low-temperature pre-baking processes in thermal evaporator chamber, equipped with contact annealing oven $\left(p<10^{-5}\right.$ atm) and alternatively, in a simple vacuum chuck, attached to the surface of standard hot-plate heater $(p<0.01 \mathrm{~atm})$. In both cases results were satisfactory.
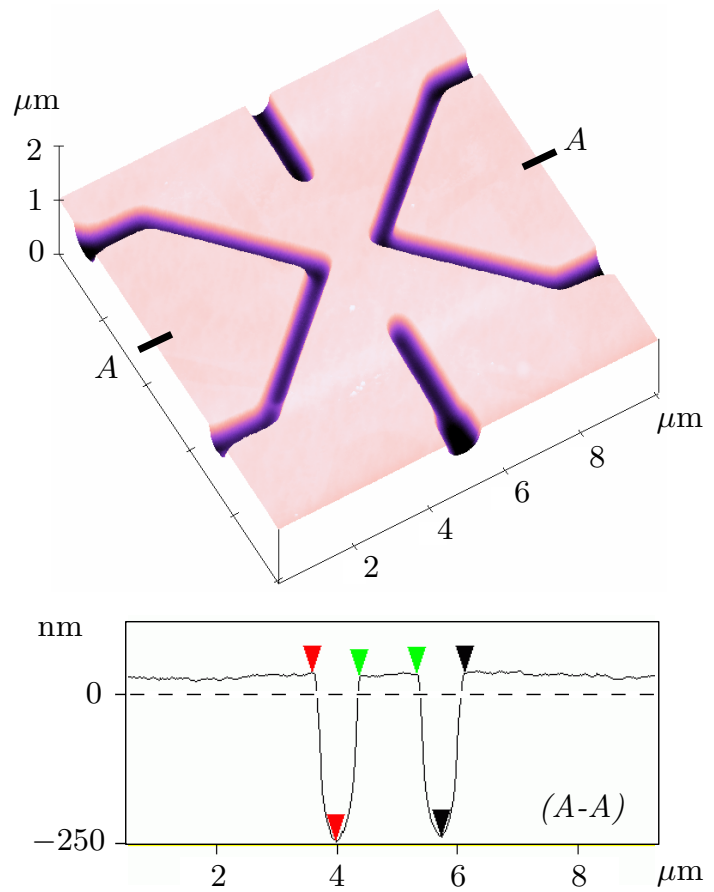

Fig. 1. Atomic force microscope image of a quantum point contact (QPC) fabricated on $\mathrm{HgTe} / \mathrm{HgCdTe}$ substrate with wet etching and PMMA as an etching mask (top). The depth profile (A-A) along the constriction (bottom).

Figure 1 shows the application of low-temperature processed PMMA as an etching mask. The pattern has been exposed with $30 \mathrm{keV}$ electron beam and size dependent dose from 180 to $300 \mu \mathrm{C} / \mathrm{cm}^{2}$. As a resist film, $9 \% 50 \mathrm{k}$ PMMA in chlorobenzene, spinned-on at $6000 \mathrm{rpm}$, has been used. Patterns have been developed by $15 \mathrm{~s}$ in water/isopropanol solution $(3: 7)$ at $21^{\circ} \mathrm{C}$. For the subsequent wet etching $0.05 \% \mathrm{Br}$ in glycol ethylene has been applied. Let us note that we have selected the smallest possible molecular weight polymer $(\approx 50000)$ to improve the adhesion at the expense of contrast and resolution power. Nevertheless, sub- $\mu \mathrm{m}$ widths of the separating grooves are easily obtained. Note also that the surface of nanostructure outside the relatively deep trenches is perfectly flat, indicating a good adhesion of resist mask to the substrate material. Otherwise, an etchant may find its way under the mask and round the mesa edges out.

Low-temperature processing has been also used for metal deposition, with the application of a so-called liftoff technique. In this case the e-beam energy has been reduced to $20 \mathrm{keV}$ and development time has been extended to $30 \mathrm{~s}$, in order to produce a stronger undercut in the developed resist. As a polymer film, high molecular weight 9\% 950k PMMA in chlorobenzene has been used, since adhesion to the substrate is not essential in this case. The results are summarized in Fig. 2. We expect that the sub- $\mu \mathrm{m}$ resolution can be easily achieved with the application of thinner PMMA films.
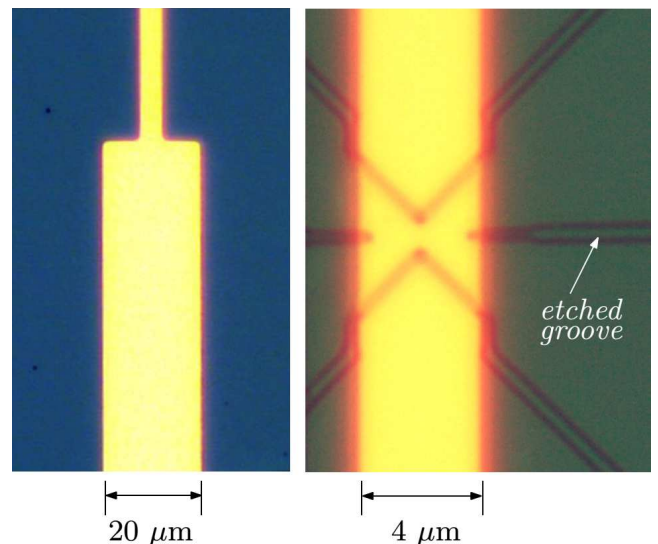

Fig. 2. Optical microscope images of $\mathrm{Ti} / \mathrm{Au}$ $(50 \AA / 150 \AA)$ metal gates fabricated with the application of lift-off technique. Right image shows the top-gate configuration when metal is evaporated directly above the etched nanostructure (on $100 \mathrm{~nm}$ thick oxide layer).

Having succeeded with PMMA we have also tested our low-temperature recipes on an alternative inorganic material, HSQ. HSQ is a negative tone resist which forms an insoluble silica-like structure when exposed to electrons [12]. With this material, sub-10 nm features have been obtained, however, at significantly higher doses than required for positive tone PMMA [9]. Figure 3 shows details of the developed test pattern consisting of $20 \mu \mathrm{m}$ long, variable distance lines, all oriented in parallel. Test pattern was exposed with $30 \mathrm{keV}$ beam energy and with dose ranging from 400 to $550 \mu \mathrm{C} / \mathrm{cm}^{2}$ on $4 \% \mathrm{HSQ}$ in MIBK, spinned-on at $6000 \mathrm{rpm}$. Standard developer, TMAH $-\left(\mathrm{CH}_{3}\right)_{4} \mathrm{NOH}$, was used for $2 \mathrm{~min}$ at $21^{\circ} \mathrm{C}$.
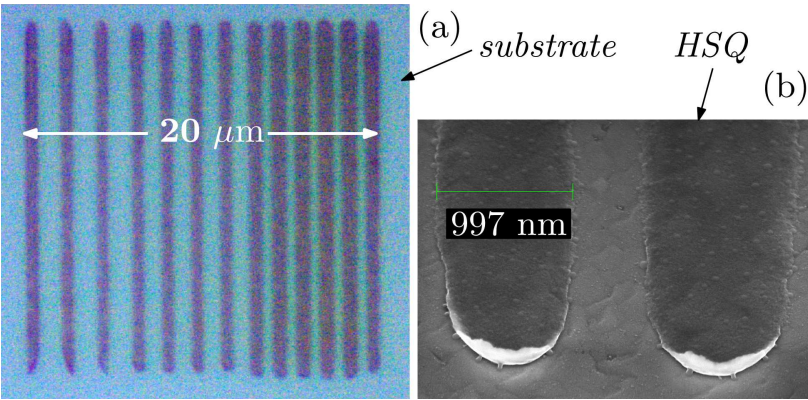

Fig. 3. Sub-micron lines formed in developed HSQ negative tone resist film. (a) Optical microscope image of $25 \times 25 \mu \mathrm{m}$ test pattern. (b) Scanning electron microscope (SEM) image of structure details.

Presented results indicate that sufficient dose has been absorbed on denser lines only, which emphasize the role of so-called proximity corrections in HSQ processing. 
Detailed SEM images of cross-linked HSQ surfaces show also that the thickness of layers is not uniform over the width and side edges are rather rough with complicated morphology. We conclude that low-temperature recipes can be applied also for HSQ, however, further studies are needed to improve the film quality.

Finally, we have addressed the problem of annealing lithographically defined electrical contacts. To avoid the overheating of a whole sample we have investigated melting of contact metal locally by exposing the selected area to the high-energy and high-current electron beam. For testing we have selected $200 \AA \mathrm{CdTe} / \mathrm{CdMgTe}$ quantum well, located $840 \AA$ below the surface. Modulation doping has been spaced by $160 \AA$ from the well barrier and sample surface has been capped with $50 \AA$ CdTe. On such substrates we have evaporated $1.2 \mu \mathrm{m}$ thick indium metal pads using thermal evaporator and substrate holder cooled by liquid nitrogen. Prior to the deposition, samples were freshed-up with $10 \mathrm{~s}$ deep in $10 \%$ water/ammonia solution. Resulting metal films were uniform and smooth with mirror-like surface. However, structures did not conduct electrically.

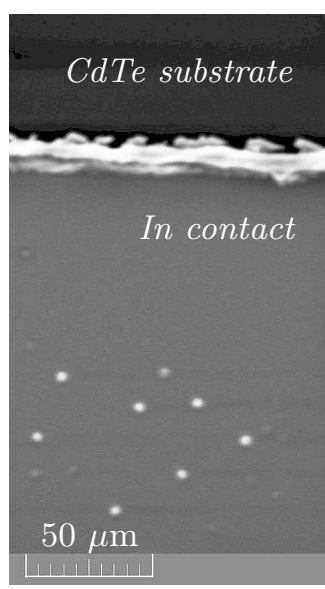

(a)

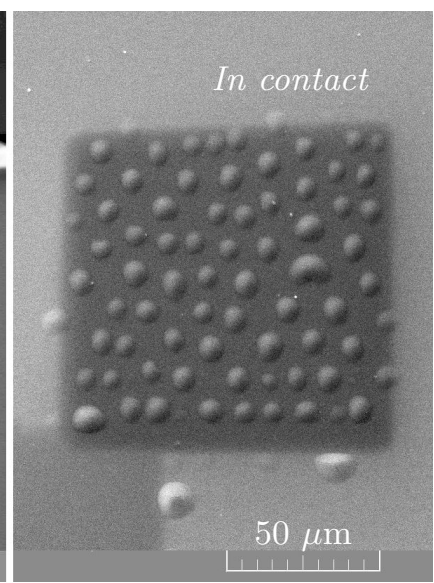

(b)
Fig. 4. SEM images of indium (In) contacts annealed locally with electrons accelerated to $V=25 \mathrm{keV}$. (a) The edge of indium layer evaporated on CdTe substrate. The lower part has been exposed with beam current $I_{\mathrm{e}}=115 \mathrm{nA}$ during $t_{\mathrm{e}}=1 \mathrm{~min}$ time interval. (b) $100 \times 100 \mu \mathrm{m}$ micro-contact area, exposed with $I_{\mathrm{e}}=600 \mathrm{nA}$ and $t_{\mathrm{e}}=5 \mathrm{~min}$ annealing.

Figure 4 shows the surface modification caused by e-beam, observed in situ after the annealing. Indium film has been melted within the scanned area but metal does not aggregate in separate droplets and contact area remains continuous. The optimal electron beam parameters and exposure times are indicated in Fig. $4 \mathrm{~b}$. After the annealing, contacts were linear (ohmic) up to voltages of $\approx 2 \mathrm{mV}$. At room temperature their resistances fell within $20-55 \mathrm{k} \Omega$ range. Such resistance drop, caused by local annealing, proves that the diffusion of indium, from contact pad towards the quantum well, has been initiated.

\section{Summary and conclusions}

To conclude, we propose low-temperature recipes for PMMA and HSQ resists to get over the incompatibilities between electron beam lithography and post-growth processing of II-VI semiconductors. The idea is based on pre-backing electron sensitive films in vacuum at $T \leq 90^{\circ} \mathrm{C}$. We have demonstrated that suggested approach shows considerable potential at the sub- $\mu \mathrm{m}$ scale, in particular for etch mask fabrication and gate metallization using the lift-off technique. Additionally, we have shown that evaporated indium films are suitable for ohmic contacts and can be melted with electron beam to promote metal diffusion locally, without overheating the rest of a sample.

\section{Acknowledgments}

The authors wish to thank M. Aleszkiewicz for AFM imaging and W. Knap for providing $\mathrm{HgTe}$ substrates. The research was partially supported by National Science Centre (Poland) under the grant DEC2012/06/A/ST3/00247 and Regional Development Program (Poland), grant WND-RPPK. 01.03.00-18-053/12.

\section{References}

[1] R. Rungsawang, F. Perez, D. Oustinov, J. Gómez, V. Kolkovsky, G. Karczewski, T. Wojtowicz, J. Madéo, N. Jukam, S. Dhillon, J. Tignon, Phys. Rev. Lett. 110, 177203 (2013).

[2] M. König, S. Wiedmann, C. Brüne, A. Roth, H. Buhmann, L.W. Molenkamp, X.-L. Qi, S.-C. Zhang, Science 318, 766 (2007).

[3] G. Grabecki, J. Wróbel, M. Czapkiewicz, L. Cywiński, S. Gierałtowska, E. Guziewicz, M. Zholudev, V. Gavrilenko, N.N. Mikhailov, S.A. Dvoretski, F. Teppe, W. Knap, T. Dietl, Phys. Rev. B 88, 165309 (2013).

[4] V. Daumer, I. Golombek, M. Gbordzoe, E.G. Novik, V. Hock, C.R. Becker, H. Buhmann, L.W. Molenkamp, Appl. Phys. Lett. 83, 1376 (2003).

[5] M. Czapkiewicz, V. Kolkovsky, P. Nowicki, M. Wiater, T. Wojciechowski, T. Wojtowicz, J. Wróbel, Phys. Rev. B 86, 165415 (2012).

[6] Y. Kim, A. Ourmazd, M. Bode, R.D. Feldman, Phys. Rev. Lett. 63, 636 (1989).

[7] S. Dvoretsky, N. Mikhailov, Y. Sidorov, V. Shvets, S. Danilov, B. Wittman, S. Ganichev, J. Electron. Mater. 39, 918 (2010).

[8] G.M. Gusev, Z.D. Kvon, O.A. Shegai, N.N. Mikhailov, S.A. Dvoretsky, J.C. Portal, Phys. Rev. B 84, 121302 (2011).

[9] M. Mohammad, M. Muhammad, S. Dew, M. Stepanova, in: Nanofabrication, Eds. M. Stepanova, S. Dew, Springer, Vienna 2012, p. 11.

[10] M.A. Mohsin, J.M. Cowie, Polymer 29, 2130 (1988).

[11] W. Chen, H. Ahmed, J. Vac. Sci. Technol. B 11, 2519 (1993)

[12] A.E. Grigorescu, C.W. Hagen, Nanotechnology 20 , 292001 (2009). 